\title{
Atomic force microscopy study of nanoindentation deformation and indentation size effect in $\mathrm{MgO}$ crystals
}

\author{
K. Sangwal ${ }^{\text {a) }}$ \\ Institute of Physics, Technical University of Lublin, ul. Nadbystrzycka 38, 20-618 Lublin, Poland \\ P. Gorostiza, J. Servat, and F. Sanz \\ Department of Physical Chemistry, University of Barcelona, Marti i Franques 1, \\ 08028 Barcelona, Spain
}

(Received 20 April 1999; accepted 28 July 1999)

\begin{abstract}
The dependences of various nanoindentation parameters, such as depth of penetration $d$, indentation diameter $a$, deformation zone radius $R$, and height $h$ of hills piled up around indents, on applied load were investigated for the initial (unrecovered) stage of indentation of the (100) cleavage faces of $\mathrm{MgO}$ crystals by square pyramidal $\mathrm{Si}$ tips for loads up to $10 \mu \mathrm{N}$ using atomic force microscopy. The experimental data are analyzed using theories of elastic and plastic deformation. The results revealed that (i) $a, R$, and $h$ linearly increase with $d$; (ii) the development of indentation size and deformation zone and the formation of hills are two different processes; (iii) the load dependence of nanohardness shows the normal indentation size effect (i.e., the hardness increases with a decrease in load); and (iv) there is an absence of plastic deformation involving the formation of slip lines around the indentations. It is found that Johnson's cavity model of elastic-plastic boundary satisfactorily explains the experimental data. The formation of hills around indentations is also consistent with a new model (i.e., indentation crater model) based on the concept of piling up of material of indentation cavity as hills.
\end{abstract}

\section{INTRODUCTION}

Indentation hardness testing is frequently used to assess the mechanical properties of crystalline and noncrystalline substances. However, despite numerous studies devoted to the understanding of the physical nature of hardness, there are several phenomena associated with indentation hardness and deformation occurring beneath and around indents that are poorly understood. Among the most widely discussed and controversial topics in the area of indentation hardness are (i) the dependence of microhardness on applied load, a phenomenon known as indentation size effect (ISE), ${ }^{1-12}$ (ii) the nature of deformation beneath and around indentations, ${ }^{1,11,13-19}$ and (iii) the mechanism responsible for the appearance of hills piled up around indents. ${ }^{17,19}$ The earlier investigations dealt with microindentation, but recently papers have also been devoted to the study of nanoindentation deformations, ${ }^{3,10,17,19,20}$ comparison of deformation in small volumes with continuum plastic and Hertzian elastic theories, ${ }^{17,19}$ and phase transitions ${ }^{15,16,20,21}$ and twinning induced by indentation deformation. ${ }^{11}$ Moreover,

\footnotetext{
a) Address all correspondence to this author. e-mail: sangwal@antenor.pol.lublin.pl
}

with an increase in load or indentation size both a decrease $^{9,10,20,22,23}$ and an increase in microhardness ${ }^{5-8,11,12}$ have been reported. These dependences are known as normal and inverse ISE, respectively.

The present paper describes an atomic force microscopy (AFM) study of (i) the interdependence of various nanoindentation parameters such as depth of penetration, indentation diameter, deformation zone size, and height of hills piled up around indents; (ii) the dependence of nanoindentation parameters on applied load; and (iii) the analysis of the experimental results using existing theories of deformation. The nanoindentation experiments were performed on the (100) cleavage faces of $\mathrm{MgO}$ crystals for loads up to $10 \mu \mathrm{N}$, and the indentation parameters were obtained from AFM images recorded immediately after deformation.

\section{EXPERIMENTAL AND ANALYSIS PROCEDURE}

$\mathrm{MgO}$ single-crystal samples were obtained by cleaving along (100) planes of starting crystals (AERE, Harwell, United Kingdom, from a batch used many years ago for etching and surface damage studies ${ }^{24,25}$ ). The crystals were kept in a desiccator for a few hours to allow them to stabilize their cleavage planes prior to indentation experiments and in situ observations by AFM. 
Nanoindentation as well as in situ AFM observations were carried out at room temperature with relative humidity of $23 \%$, using a commercial Nanoscope III manufactured by Digital Instruments, Santa Barbara, CA. The same AFM tips were used as indenters as well as surface probes, which enabled the selection of relatively large flat regions free of cleavage steps for indentation experiments and to follow in situ the appearance and evolution of each indentation individually. Indents were imaged within 5-10 s after being made, with a "time resolution" of about $30 \mathrm{~s}$ between successive images. The AFM was operated in contact mode using silicon microfabricated tips (Nanoprobes, Wetzlar-Blankenfeld, Germany).

The cleaved samples were mounted in the nanoscope head in such a manner that the indents were always scanned in the [100] direction. Indentations were made in regions selected from $15 \times 15 \mu \mathrm{m}$ areas. The regions for indentations were chosen with the criteria that there were relatively smooth large areas of the cleaved surface, free of visible cleavage steps, as observed optically. In such areas large flat strips with cleavage steps running approximately along the [010] direction could easily be located as reference for indentation. This procedure for mounting the sample was adopted in view of the fact that the hardness of $\mathrm{MgO}$ crystals is anisotropic. ${ }^{13,26}$

In contrast to tapping mode in which a constant test load cannot be applied uninterruptedly, penetration of a tip into a specimen surface in the contact mode is well suited for indentation hardness studies because experimental parameters like test load, indentation time, and indenter penetration rate can be specified. Moreover, the resolution of surface features is better in the contact mode. According to the specifications provided by the manufacturer, the Si tips were square pyramidal in shape and had spring constants of 20-100 N/m, resonance frequencies of $200-400 \mathrm{kHz}$, tip radii of $5-10 \mathrm{~nm}$, wedge inclination angles of $18^{\circ}$ (side) and $25^{\circ}$ (front), and a cantilever length of $125 \mu \mathrm{m}$. Conventional silicon nitride contact AFM tips were found to be unsuccessful in indenting the $\mathrm{MgO}$ cleavages due to either their low spring constant values (up to $0.6 \mathrm{~N} / \mathrm{m}$ ) or their large tip apex radius (between 20 and $40 \mathrm{~nm}$ ).

Each indentation experiment entailed the making of an indent and subsequent in situ observation of its evolution. Depending on the initial size of the indent and the type of information expected, the observation lasted for durations from several minutes to several hours. A number of different tips of various resonance frequencies and spring constants were used. Therefore, before each indentation experiment by a particular tip, indentation parameters (i.e., cantilever force constant and penetration depth) were calibrated.

In order to make an indentation and to follow subsequent real-time evolution, an image of the surface was first acquired at the minimum force setpoint. During im- aging, the lateral scanning was then stopped at a selected position on the surface and the feedback control disconnected. The tip was then driven into the selected position on the surface of the sample at a particular rate by the piezoelectric driver, thus allowing it to indent the surface for a particular dwell time. Immediately after the act of indentation, the imaging conditions were restored.

A threshold force was found to be necessary to make an indent. Since in our experiments several different tips were used for acquiring images and making indents, the threshold load was determined before every indentation experiment. Tips which gave threshold pressures (calculated from applied load $P$ and nominal tip radius $R_{\text {tip }}=$ $10 \mathrm{~nm}$ ) exceeding $3 \mathrm{GPa}$ were not used. In these cases it was believed that the tip radius was much larger than that given in manufacturer's tip specifications. The values of force pulses $P$ corresponding to each indentation were calculated by multiplying the approach distance $X$ given by piezoelectric and the tip spring constant $K$ calculated from its vibration frequency $f$. The spring constant $K$ for a tip was calculated from the relation ${ }^{27} K=0.24 m_{\mathrm{c}} \omega_{0}{ }^{2}$, where the cantilever mass $m_{\mathrm{c}}$, estimated from manufacturer's tip specifications, is equal to $3.38 \times 10^{-11} \mathrm{~kg}$ and the resonance frequency $\omega_{0}=2 \pi f$. Indentations were made with a constant penetration rate of $10 \mu \mathrm{m} / \mathrm{s}$ and a dwell time of $1 \mathrm{~s}$ at peak load.

For some tips the threshold load was also determined for two successive indentations to check whether a particular tip had undergone deformation. The values of load thresholds for successive indentation were found to remain essentially unchanged, suggesting that the tips are not deformed by indentation.

In the present work, only the first indents scanned immediately after indentation were considered for analysis. Although some "recovery" could have occurred during the 5-10 s elapsed between the act of indentation and the imaging, this time delay is negligible in comparison with the time delay occurring in ex situ experiments involving transfer of specimens, indented separately by a nanoindenter ${ }^{17}$ or a microindenter,${ }^{11}$ for AFM examination. Therefore, in contrast to the latter case in which a large recovery of indents, as noted from their flat bottoms, already occurs during the time of transfer, our procedure ensures the examination of essentially unrecovered indents. It is worth noting that our procedure is very similar to that recently followed by Lilleodden et al. ${ }^{19}$

The indentation impressions were circular at low pressures of up to about $5 \mathrm{GPa}$, but they tended to acquire a somewhat asymmetric four-sided shape at higher loads. The asymmetry became pronounced when indentations were made on poorly mounted samples in which a sample drift usually occurred during indentation and imaging. Typical examples of indents produced at two different loads are illustrated in Fig. 1. As seen from this figure, an indent has a region of protruded material 
around it and the size of this region increases with the applied load. Moreover, one can clearly see parallel elementary cleavage steps of height $0.29 \mathrm{~nm}$, but no slip lines are discerned around the indentations.

Nanoindentation deformation data were obtained from the recorded images of indents. For an indent corresponding to a particular load, the penetration depth $d$ of the indent, its diameter $a$, the radius $R$ of deformation zone, and the height $h$ of pile up around it were measured. Figure 2 shows the profile and the three-dimen-

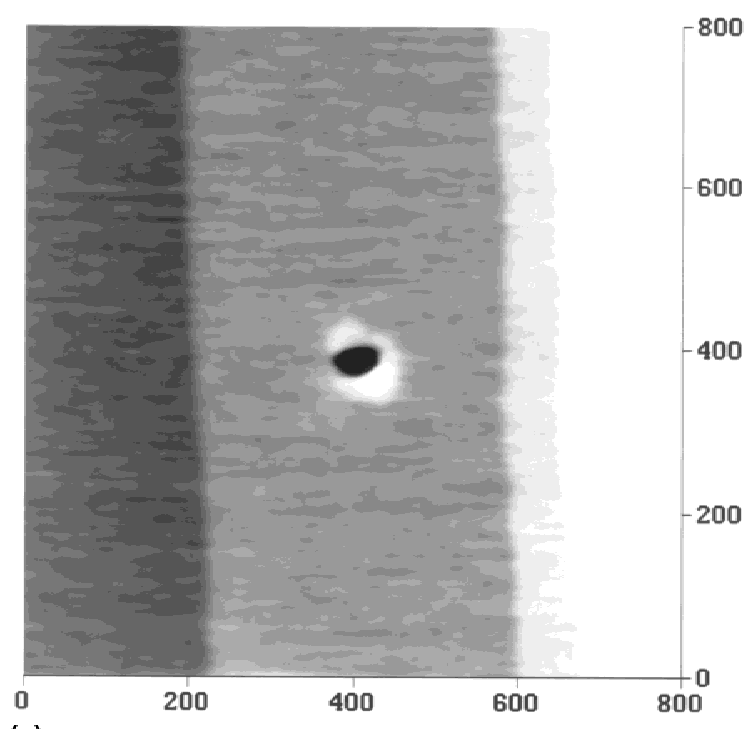

(a)

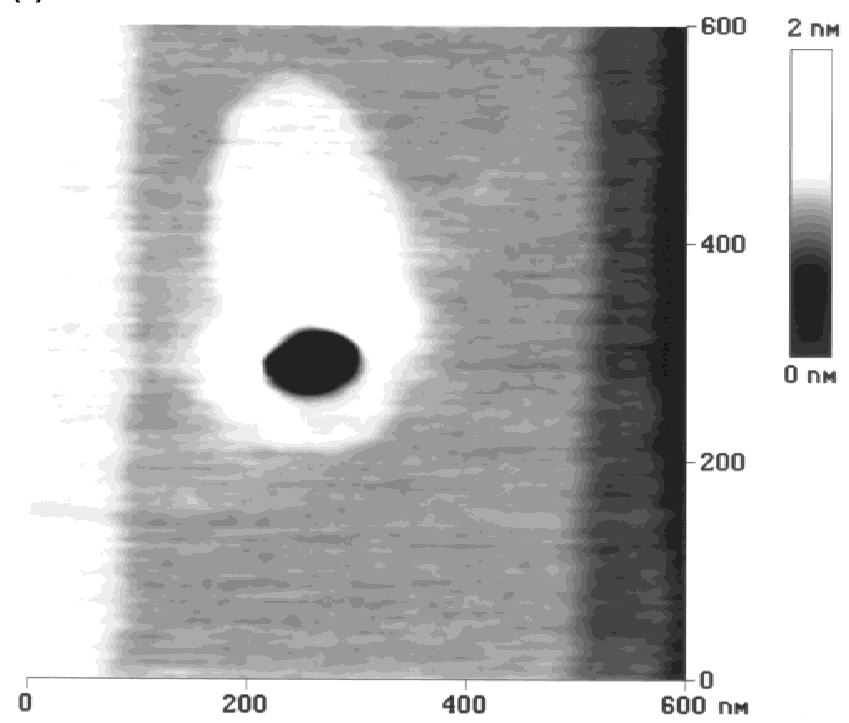

(b)

FIG. 1. Typical examples of indents produced at two different loads: (a) $1.12 \mu \mathrm{N}$, (b) $2.22 \mu \mathrm{N}$. It may be noted that an indent has a region of protruded material around it. With reference to vertical elementary cleavage steps of height $0.29 \mathrm{~nm}$ it may be seen that no slip traces are produced around the indentation. In the upper part of (b) the large gray area on the cleavage surface shows material "spilt out" from the indentation hill. The height of the layer of this spilt out material is also $0.29 \mathrm{~nm}$. sional view of an indent made with an indentation load of $1.78 \mu \mathrm{N}$. The various parameters considered for analysis are schematically illustrated in Fig. 3. Each parameter was measured twice at the deepest level of the indent, keeping the tracer parallel to the $x$ and $y$ directions.

The shape and size of features observed from AFM measurements are always convoluted with the shape of the probe. In the present case it may be believed that the convolution effect does not affect $h$ and that $R$ and $a$ are measured accurately because their dimensions are much larger than the tip radius $R_{\text {tip }}$. One may suspect that the measured $d$ values may have systematic errors. However, according to our estimates these measured $d$ values are not altered by more than $5 \%$ due to the convolution effect. The linear dependences of $R, a$, and $h$ on $d$ in the entire range lends support to this inference.

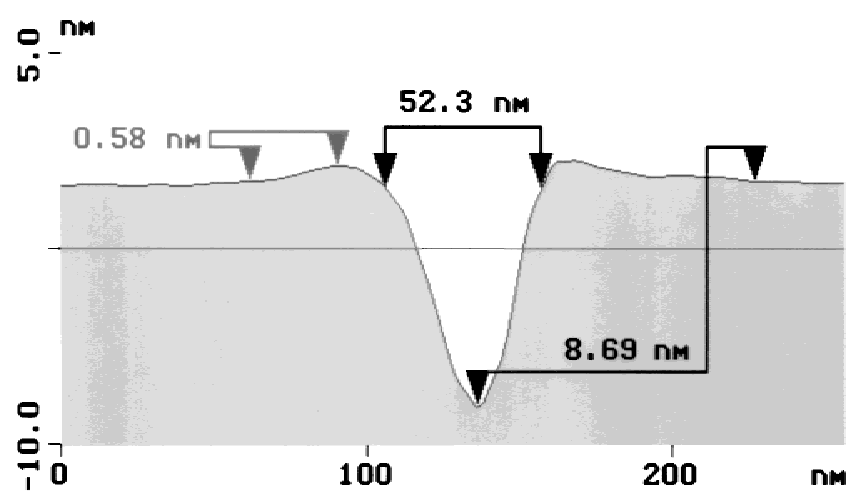

(a)

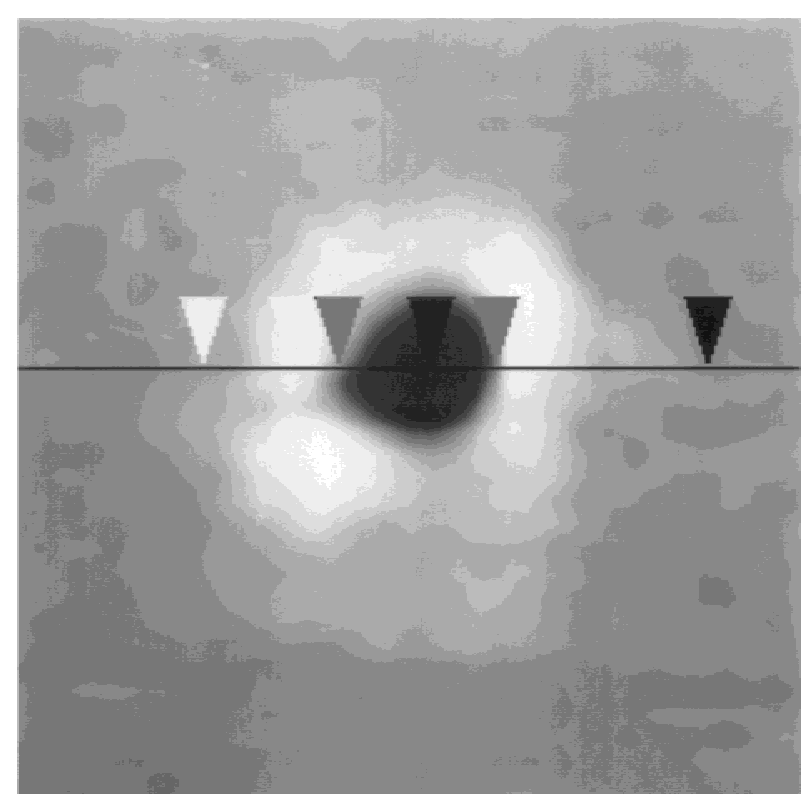

(b)

FIG. 2. Profile of an indent made at a load of $1.78 \mu \mathrm{N}$ : (a) indentation profile showing measurements of indentation depth (dark markers), indent diameter (gray markers), and hill height around indents (white markers); (b) image of indent. Note that a hill is not usually symmetrically developed around the indent, as seen from (b). 
For data analysis, an arithmetic average was taken for measured depth $d$ while geometric averages were taken for $a, R$, and $h$. In the latter case, especially for $a$ and $R$, arithmetic averages, which were essentially not much different from the geometric averages, could also have been used. However, in view of large differences in the height of hills around the indents along $x$ and $y$ directions $\left(h_{y} / h_{x}\right.$ up to 7$)$ at high loads, it was thought that perhaps it would be more reasonable to use geometric averages in the analysis of the data.

\section{RELATIONSHIP BETWEEN PENETRATION DEPTH AND OTHER INDENTATION PARAMETERS}

The relationships between the measured indenter penetration depth $d$, the indentation diameter $a$, the deformation zone radius $R$, and the average height $h$ of

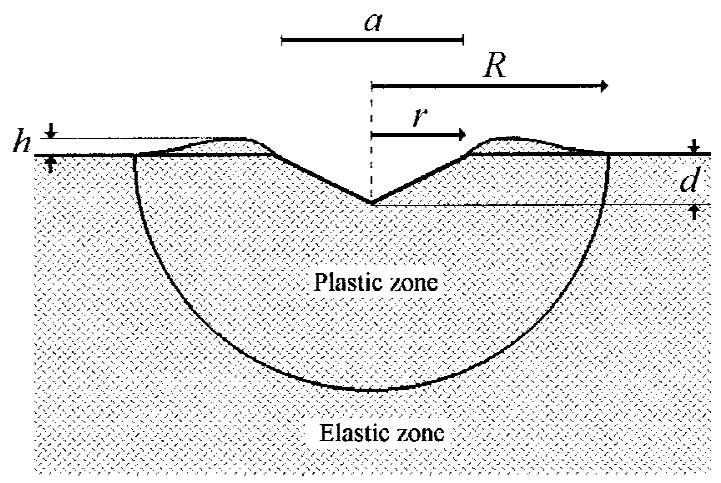

FIG. 3. Schematic illustration of various parameters considered for analysis and definition of elastic and plastic zones. In the range of load used during indentation of $\mathrm{MgO}$ here, no evidence of plastic deformation, as deduced from lack of slip traces around the indents, was found. Therefore, in view of the lack of more information about the plastic zone, the term deformation zone has also been used in the text.

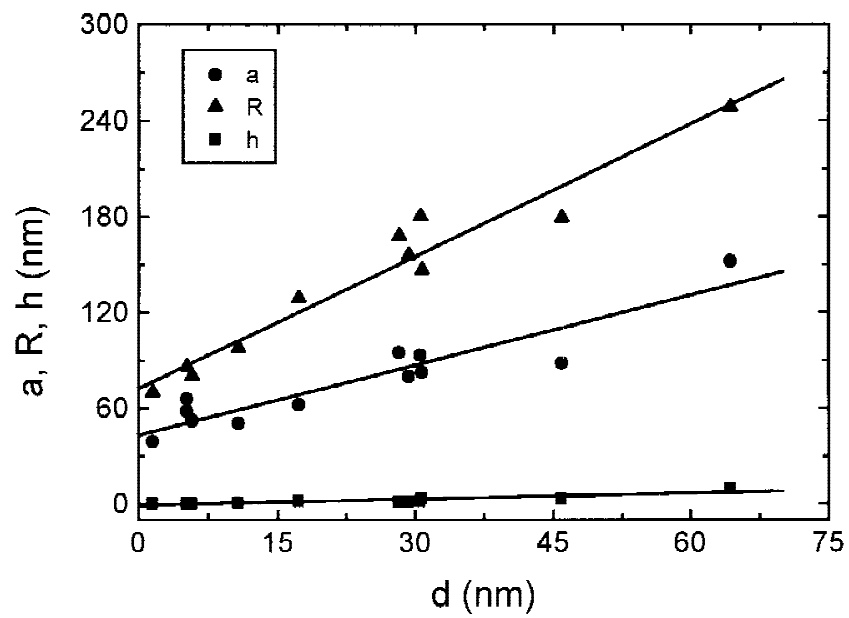

FIG. 4. Dependence of indentation diameter $a$, radius $R$ of deformation zone, and average height $h$ of indentation hills on the depth of penetration $d$. Note positive values of intercepts for $a(d)$ and $R(d)$ plots. indentation hills are shown in Fig. 4. It is obvious from the figure that these relations may be expressed by the equation

$$
y=y_{0}+k d=k\left(d_{0}+d\right)=k d^{*},
$$

where $k$ is the ratio of $y$ to $d$ for $y>y_{0}, d_{0}$ is the threshold value of $d$ beyond which the linear relationships are valid, and $d^{*}=d_{0}+d$ is the corrected indentation depth. It was also found that $h$ linearly increases with $a$ for $a$ > $a_{0}$. The values of different parameters are given in Table I.

As seen from Table I, the values of $d_{0}$ obtained from $R(d)$ and $a(d)$ plots are 26.6 and $26.3 \mathrm{~nm}$, while that obtained from the $h(d)$ plot is practically zero. Using the value of $a_{0}$ and the corresponding $d_{0}$, one obtains the value of the semiapical angle $\beta=\tan ^{-1}\left(a_{0} / 2 d_{0}\right)=36^{\circ}$ $\pm 3^{\circ}$. This value of the angle $\beta$ is somewhat higher than that given in the catalog of tips. A higher value of $\beta$ from the indents and the observation of threshold $d_{0}$ are due to elastic recovery of the indent as load is released.

It is interesting to note that the ratio $R_{0} / r_{0}$ (also the ratio $R / r$ ) of the radius of deformation zones to that of indents is 3.3 (i.e., $R / a=1.67$ ). This value is comparable with the ratio of size of dislocated region and diagonal of Vickers indenter in the range of microindentations into the (100) face of $\mathrm{Fe}-3 \% \mathrm{Si}^{17}$ However, in contrast to indentation deformation involving dislocations in $\mathrm{Fe}-3 \%$ $\mathrm{Si}$, no slip lines indicating the generation of dislocations on the surface surrounding indents were observed.

The above results reveal that, although $a, R$, and $h$ linearly increase with $d$, there are two different processes involved during indentation. The first is associated with the development of indentation size and deformation zone, while the second is associated with the formation of hills which occurs as soon as the threshold $d_{0}$ is attained. Moreover, from the $R(d)$ and $a(d)$ relations, it may be seen that the deformation zone sets in (i.e., $R \geqslant 0$ ) when $a \geqslant-5.4 \mathrm{~nm}$. This $a$ value probably originates from measurement errors.

It is interesting to note that, although the elastic modulus $E$ of $\mathrm{Si}$ is lower than that of $\mathrm{MgO}$, Si tips produce indentation imprints on the (100) face of $\mathrm{MgO}$. From a consideration of the values of the elastic moduli $[E(\mathrm{Si})=$ $166 \mathrm{GPa}$ and $\left.E(\mathrm{MgO})=286 \mathrm{GPa}^{28}\right]$ it is expected that Si tips undergo plastic deformation which will lead to an increase in the tip radius during successive indentations. In our experiments no such changes in the radius of a tip

TABLE I. Values of $y_{0}, k$, and $d_{0}$ of Eq. (1).

\begin{tabular}{ccccc}
\hline \hline Dependence & $\begin{array}{c}\text { Threshold } \\
\text { parameter }(\mathrm{nm})\end{array}$ & $\begin{array}{c}\text { Slope } k \\
\left(\mathrm{~nm}^{-1}\right)\end{array}$ & $d_{0}(\mathrm{~nm})$ & $\begin{array}{c}\text { Correlation } \\
\text { coefficient }\end{array}$ \\
\hline$a(d)$ & $a_{0}=43.36$ & 1.46 & 29.68 & 0.9292 \\
$R(d)$ & $R_{0}=72.60$ & 3.51 & 26.34 & 0.9725 \\
$h(d)$ & $h_{0}=0.066$ & 0.071 & 0.93 & 0.8368 \\
\hline \hline
\end{tabular}


were recognized from the load threshold checked before and after indentation. To explain this observation we consider the reduced tip-crystal elastic modulus, given by

$$
\frac{1}{E^{*}}=\frac{\left(1-v_{\mathrm{m}}^{2}\right)}{E_{\mathrm{m}}}+\frac{\left(1-v_{\mathrm{i}}^{2}\right)}{E_{\mathrm{i}}}
$$

where $E$ denotes the elastic modulus, $v$ is Poisson's ratio, and the subscripts $\mathrm{m}$ and $\mathrm{i}$ denote the material and the indenter, respectively. Using the values of $E(\mathrm{Si})$ and $E(\mathrm{MgO})$ given above and taking $v_{\mathrm{m}}=\nu_{\mathrm{i}}=0.3$, one obtains $E^{*}=115 \mathrm{GPa}$. This reduced $E^{*}$ is lower than the values of both $E(\mathrm{Si})$ and $E(\mathrm{MgO})$. This suggests that the ability of indentation of a tip of elastic modulus $E$ lower than that of the crystal is determined by the reduced tip-crystal elastic modulus $E^{*}$.

\section{LOAD DEPENDENCE OF PENETRATION DEPTH AND OTHER PARAMETERS}

\section{A. Load versus $a^{2}, R^{2}$, and $d^{\star 2}$ relations}

For rigid plastic materials, Tabor ${ }^{29,30}$ gives a relation between indentation load $P$ and indentation diameter $a$ for a conical or pyramidal indenter in the form

$$
P=H_{\mathrm{M}} \pi a^{2} / 4=0.785 H_{\mathrm{M}} a^{2},
$$

where $H_{\mathrm{M}}$ is the Mayer hardness of the material. In Eq. 3 it is assumed that $H \approx 3 Y$, where $Y$ is the yield stress of the material. On the other hand, for an elastic material subjected to conical punching Sneddon gives the relation $^{4,30}$

$P=\frac{2 E^{*} d^{2}}{\pi\left(1-v^{2}\right)} \tan \beta=0.506 E^{*} d^{*^{2}}=0.237 E^{*} a^{2}$

where $v$ is Poisson's ratio, $\beta$ is the semiapical angle of the cone, $E^{*}$ is the reduced elastic modulus of the system, and $a / d^{*}=a /\left(d+d_{0}\right)=1.461$ (see Table I). In this equation we have substituted the values of $\beta=36^{\circ}$ and $v=0.3$.

According to Johnson's cavity model, ${ }^{17,19,31}$ the relation between applied load $P$ and deformation zone radius $R$ is given by

$$
P=2 \pi Y R^{2} / 3=2.1 Y R^{2},
$$

where $Y$ is the yield point of the material under investigation.

Figure 5 shows the plots of $P$ against $a^{2}$ and $R^{2}$, while the different parameters calculated from the above equations are given in Table II. It may be seen from the figure that, as expected from the three relations Eqs. (3)-(5), $P$ is linearly related with $a^{2}$ and $R^{2}$.

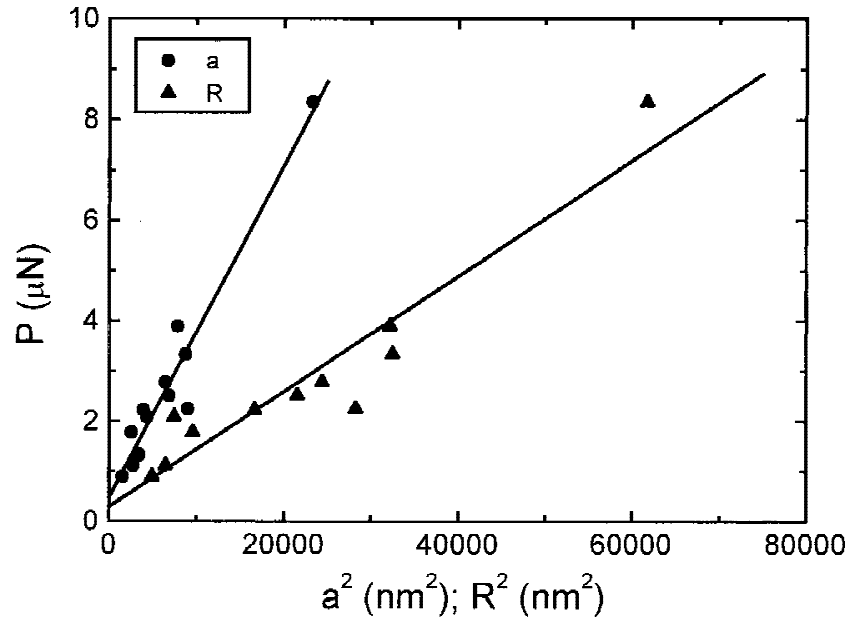

FIG. 5. Plots of the dependence of $P$ on $a^{2}$ and $R^{2}$, according to Eqs. (3)-(5): curve a, Tabor's and Sneddon's models; curve R, Johnson's cavity model.

Table II shows that the Meyer hardness $H_{\mathrm{M}}$ obtained from Tabor's relation is lower by a factor of 10-20 than the literature value, ${ }^{5,13,26}$ which lies between 4 and $8 \mathrm{GPa}$. The value of $E^{*}$ predicted by Sneddon's model for an elastic material is lower than the reduced value by a factor of 80. However, the value of the yield point $Y=$ $55 \mathrm{MPa}$ obtained from Johnson's cavity model is close to the value of $80 \mathrm{MPa}$ reported in the literature. ${ }^{32}$ From these results of the analysis it may be concluded that Tabor's model for perfect rigid plastic solids and Sneddon's model for perfect elastic materials do not explain the nanoindentation data on $\mathrm{MgO}$ crystals and that Johnson's cavity model reasonably well describes the experimental $P(R)$ data.

It is interesting to note that, despite the fact that Tabor's and Sneddon's models are not valid, the values of $a_{0}$ and $d_{0}$ given in Table II are in very good agreement with those deduced from the plots of Fig. 1 (see Table I). However, although Johnson's cavity model reasonably describes the experimental $P(R)$ data, the value of $R_{0}$ is about $2 / 3$ of the value obtained from the $R(d)$ plot (see Fig. 1 and Table I). Moreover, from the experimental $P(R)$ data Johnson's cavity model gives the value of yield stress $Y$ which is lower than the experimental value by a factor of about 1.5. These discrepancies may arise due to two reasons: (i) the outer boundary of indentation hills, from which $R$ values were measured, probably does not represent the exact boundary of the deformed zone around an indenter and is lower by a factor of about 1.5 , and (ii) assuming that Johnson's cavity model correctly predicts the yield stress $Y$, the literature $Y$ value is higher than the true value by a factor of 1.45 .

\section{B. Hertzian theory of elastic contacts}

Following Tabor ${ }^{29,30}$ and Hammond and Armstrong, ${ }^{33}$ the relation between hardness stress $\sigma_{\mathrm{H}}=P /\left(\pi a^{2} / 4\right)$ and 
TABLE II. Values of intercept, slope, threshold, and estimated values of stress parameters.

\begin{tabular}{cccccc}
\hline \hline Dependence & $\begin{array}{c}\text { Intercept } \\
(\mu \mathrm{N})\end{array}$ & $\begin{array}{c}\text { Slope } \\
(\mathrm{MPa})\end{array}$ & $\begin{array}{c}\text { Threshold } \\
\text { parameter }(\mathrm{nm})\end{array}$ & $\begin{array}{c}\text { Correlation } \\
\text { coefficient }\end{array}$ & $\begin{array}{c}\text { Estimated stress } \\
\text { parameter }(\mathrm{MPa})\end{array}$ \\
\hline$P\left(a^{2}\right)$ & 0.491 & 330.55 & $a_{0}=38.54$ & 0.9659 & $H_{\mathrm{M}}=420$ \\
$P\left(R^{2}\right)$ & 0.267 & 115.27 & $R_{0}=48.16$ & 0.9150 & $Y=55$ \\
\hline \hline
\end{tabular}

hardness strain $a / 2 R_{\text {tip }}$, according to the classical Hertzian theory, may be given by

$$
\sigma_{\mathrm{H}}=\frac{4}{3 \pi} E^{*}\left(a / 2 R_{\mathrm{tip}}\right)
$$

where the reduced elastic modulus $E^{*}$ is given by Eq. (2).

Figure 6(a) shows the experimental data of the dependence of $\sigma_{\mathrm{H}}$ on $a / 2 R_{\text {tip }}$ and the predicted dependence according to Eq. (6) for two values of $E^{* / 1000}=$ $115 \mathrm{MPa}$ and $E^{* / 100}=1.15 \mathrm{GPa}$. It may be seen from the figure that the experimental $\sigma_{\mathrm{H}}$ values lie between the two curves with $E^{*}$ values which are lower by a factor of $10^{2}-10^{3}$. Moreover, in contrast to a predicted linear increase in $\sigma_{\mathrm{H}}$ with $a / 2 R_{\text {tip }}$ by Eq. (6), the experimental $\sigma_{\mathrm{H}}$ values steadily decrease with an increase $a / 2 R_{\text {tip }}$.

According to the Hertzian theory for perfect elastic contacts with a spherical tip of radius $R_{\text {tip }}$, the relation between load $P$ and penetration depth $d^{*}$ below the yield point is given by ${ }^{31}$

$$
P=4 / 3 E^{*} R_{\mathrm{tip}}^{1 / 2} d_{\mathrm{el}}^{3 / 2},
$$

where $d_{\mathrm{el}}=d^{*}$ is the penetration depth for a perfect elastic contact.

Figure 6(b) shows the plot of the dependence of $P$ on $d_{\mathrm{el}}^{3 / 2}$. The figure shows that the data are well described by relation (7). The slope of the plot is $4.86 \mathrm{~N} / \mathrm{m}^{3 / 2}$ which gives $E^{*}=36,450 \mathrm{GPa}$ for the tip radius $R_{\text {tip }}=10 \mathrm{~nm}$. This value is higher than the actual value of $E^{*}=$ $115 \mathrm{GPa}$ by a factor of 320 .

Thus, it may be concluded that the Hertzian theory does not explain the experimental results satisfactorily and that there is a large disgreement in the estimated values of $E^{*}$.

\section{Indentation size effect}

From Fig. 6(a) it may be seen that nanohardness $H=$ $\sigma_{\mathrm{H}}$ decreases with an increase in indentation size $a$ (or indentation depth $d^{*}$ and load $P$ ). In order to explain this type of load dependence of microhardness, several approaches have been used in the literature: Hays and Kendall's approach involving a threshold load to induce plastic deformation, ${ }^{34}$ the elastic/plastic deformation respond (EPD) model of Bull et al., ${ }^{5}$ the proportional specimen resistance (PSR) model of Li and Bradt, ${ }^{6-8}$ the geometrical scaling model of Ma and Clarke, ${ }^{11}$ and the strain gradient plasticity model. ${ }^{11,35,36}$ Most of these models involve plastic deformation during indentation. The geometrical scaling and the proportional specimen resistance models are essentially the same. In these models the indenter edges determine the load dependence of hardness, and nothing is implied about the presence or generation of dislocations in the specimen during the penetration of an indenter into its surface. The strain gradient plasticity theory is for plastically deforming materials and is based on the presence of dislocations.

Analysis of experimental data for silver showed ${ }^{11}$ that the strain gradient plasticity theory and the geometrical scaling model give similar fits of the data. Similarly, analysis of experimental data on $\mathrm{PbS}, \mathrm{BaFCl}$, and $\mathrm{ZnSnAs}_{2}$ revealed $^{10}$ that the EPD and the PSR models give similar fits. Thus, it may be concluded that the EPD, PSR, geometrical scaling model, and strain gradient theory essentially give similar results and that the presence of dislocations or the occurrence of slip and the motion of dislocations along slip bands is not essential for these models to describe the load dependence of hardness.

In view of the fact that slip traces were not observed on the (100) face of $\mathrm{MgO}$, we analyzed our data for the size dependence of $\sigma_{\mathrm{H}}$ by following the general equation of models which do not involve the generation or presence of dislocations. This equation is of the following form: ${ }^{6-8,11}$

$$
P / a^{2}=A_{1}+B_{1} / a,
$$

where the first term $A_{1}$ denotes the load-independent part of hardness while the second term $B_{1} / a$ represents the load-dependent hardness and $A_{1}$ and $B_{1}$ are material constants. For a spherical indenter, $\sigma_{\mathrm{HO}}=4 A_{1} / \pi$ is the loadindependent hardness. Figure 7 illustrates the plot of $\sigma_{\mathrm{H}}=4 P / \pi a^{2}$ against $1 / a$, which yields $\sigma_{\mathrm{H} 0}=294 \mathrm{MPa}$. In comparison with the reported microhardness, this value is very low.

The hardness stresses involved during the nanoindentation of the (100) face of $\mathrm{MgO}$ lie between 300 and $900 \mathrm{MPa}$. These stresses are higher by a factor of 4-12 than the reported yield point $Y^{32}$ and are lower by a factor of 10-25 than the reported microhardness of MgO crystals. ${ }^{5,13,26}$ This means that our nanoindentation stresses lie between stresses involved in inducing plastic deformation and producing microindentations accompanied by plastic deformation (slip lines). However, since we did not observe slip traces due to the generation of dis- 

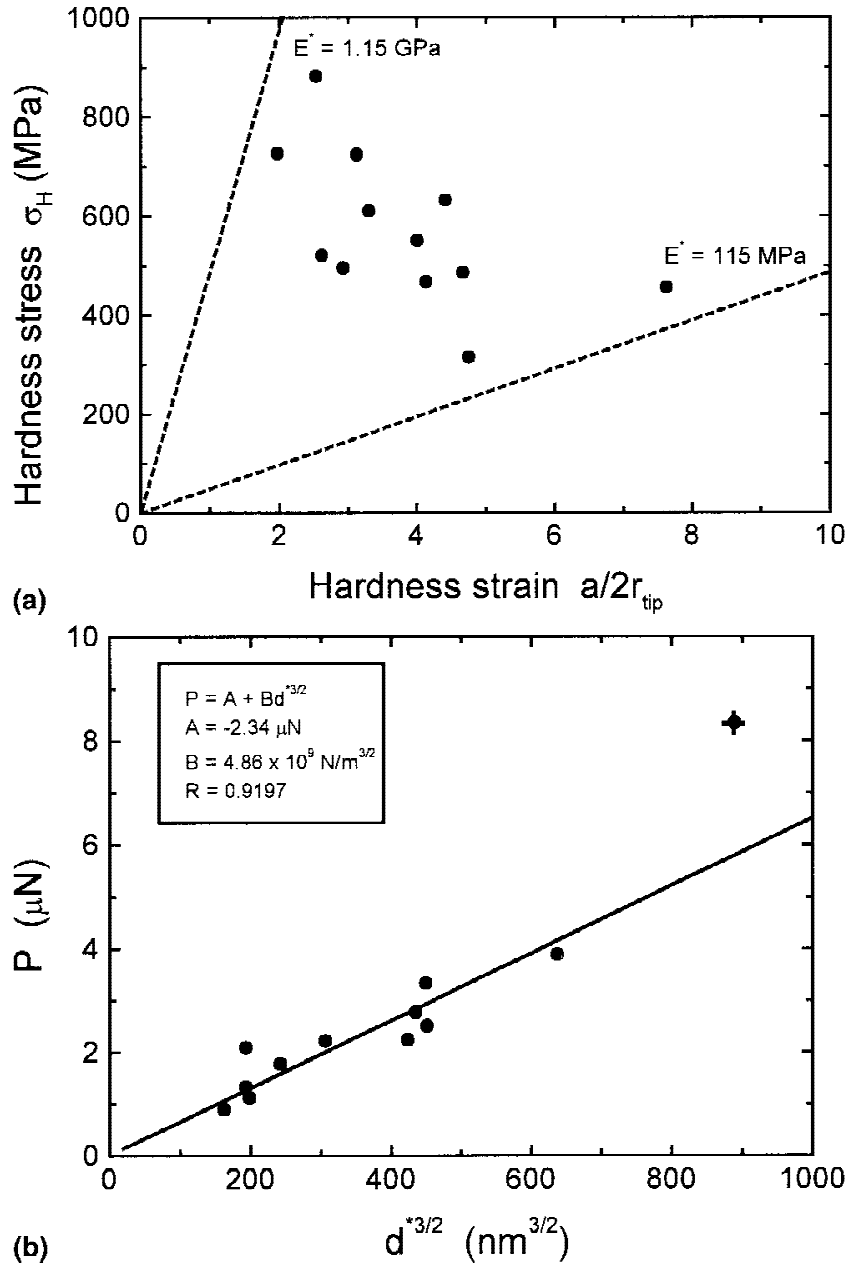

FIG. 6. Verification of Hertzian theory from plots of the dependence of (a) hardness stress $\sigma_{\mathrm{H}}=P /\left(\pi a^{2} / 4\right)$ on hardness strain $a / 2 R_{\text {tip }}$ [Eq. (6)] and (b) load $P$ on $d^{3 / 2}$ [Eq. (7)]. In (b) the point indicated by the + symbol was not taken into consideration during analysis.

locations, it may be concluded that the generation of dislocations is not essential for the observation of ISE during nanoindentation. The large discrepancy between nano- and microhardness is due to the assumption that the ratio of hardness $H$ to yield stress $Y$ is 3 [for example, in Tabor's model for rigid plastic solids; Eq. (3)]. For most crystals $\mathrm{H} / Y \approx 355^{30,37}$ There is one more difference between hardnesses determined from nano- and microindentations. Our in situ examination of nanoindentations revealed that they are recovered after sufficiently long periods of time and the recovery time depends on the value of the initially applied indentation load. ${ }^{38}$ In contrast to this, microindentation always produces permanent deformation (plastic deformation and cracks) and strictly follows the definition of hardness.

It is usually found that, in Meyer's law (i.e., $H_{\mathrm{M}}=$ $A_{0} a^{n}$, where $A_{0}$ is a material constant), the exponent $n<2$ and $n>2$ for normal and reverse ISE, respectively. In the case of normal ISE, the value of $n<2$ has been sug-

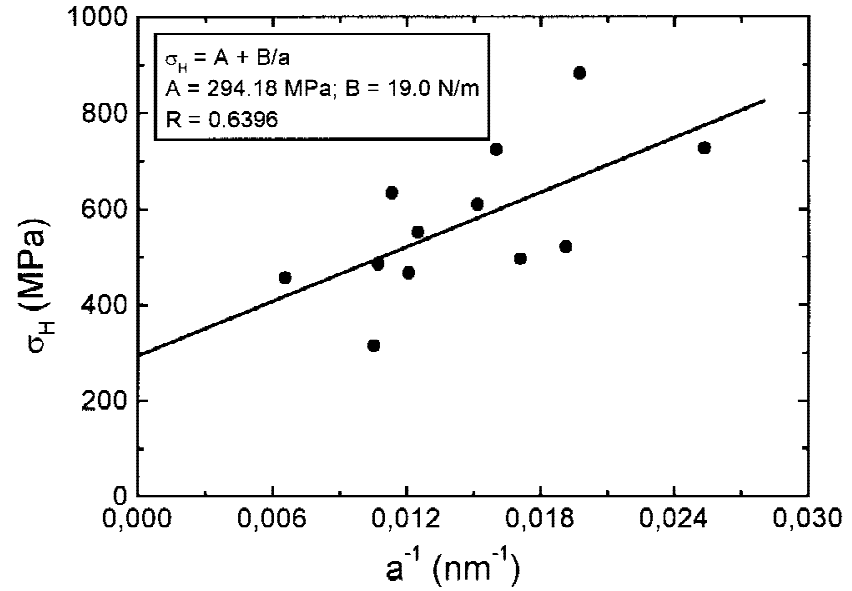

FIG. 7. Dependence of hardness stress $\sigma_{\mathrm{H}}=4 P / \pi a^{2}$ on inverse of indentation diameter $a$.

gested $^{3,39,40}$ to be associated with work-hardening characteristics of the material where generation, mobility, and multiplications of dislocations are involved. A possible cause for the increase in hardness at which $n \neq 2$ is a decreasing size of the dislocation source. ${ }^{3}$ Apart from the elastic stage in which Hooke's law holds (i.e., ratio of stress to strain is equal to Young's modulus E), the stress-strain behavior of plastic crystals shows at least four stages with differing work-hardening coefficients $\theta .{ }^{41}$ The hardening coefficients in stages I and II are constant and are associated with easy glide and multiple slip, respectively. However, in stages III and IV the hardening coefficients decrease with increasing strain. These latter stages of decreasing work hardening involve cross slip and redistribution of defects (dislocations and point defects) produced during deformation into configurations with lower energy (polygonization and annihilation of dislocations).

Here we are not concerned with the strain-independent hardening coefficients of stages I and II. However, we consider the strain-dependent hardening coefficients of stages III and IV, because there appears a similarity between stages III and IV of stress-strain curves and the dependence of hardness stress on hardness strain. Following Gil Sevillano, ${ }^{41}$ the dependence of coefficient $\theta=\sigma_{\mathrm{H}} /\left(a / 2 R_{\text {tip }}\right)$ on hardness stress $\sigma_{\mathrm{H}}$ may be given by

$$
\theta=\theta_{0}\left(1-\sigma_{\mathrm{H}} / \sigma_{\mathrm{Hs}}\right),
$$

where $\theta_{0}$ corresponds to the hardening coefficient at zero stress and $\sigma_{\mathrm{Hs}}$ is a saturation stress.

Figure 8 shows the plot of the dependence of $\theta$ on $\sigma_{\mathrm{H}}$. From the plot one obtains $\theta_{0}=-147.7 \mathrm{MPa}$ and $\sigma_{\mathrm{Hs}}=$ $260.4 \mathrm{MPa}$. In contrast to positive values of $\theta_{0}$ observed in stress-strain curves where strain hardening takes place, in the present case $\theta_{0}$ is negative. The negative value of $\theta_{0}$ means that softening occurs during normal ISE. The value of $\sigma_{\mathrm{HO}}$ is comparable with the value of load-independent hardness $\sigma_{\mathrm{H}}$ at $a \rightarrow \infty$ (see Fig. 7). One 


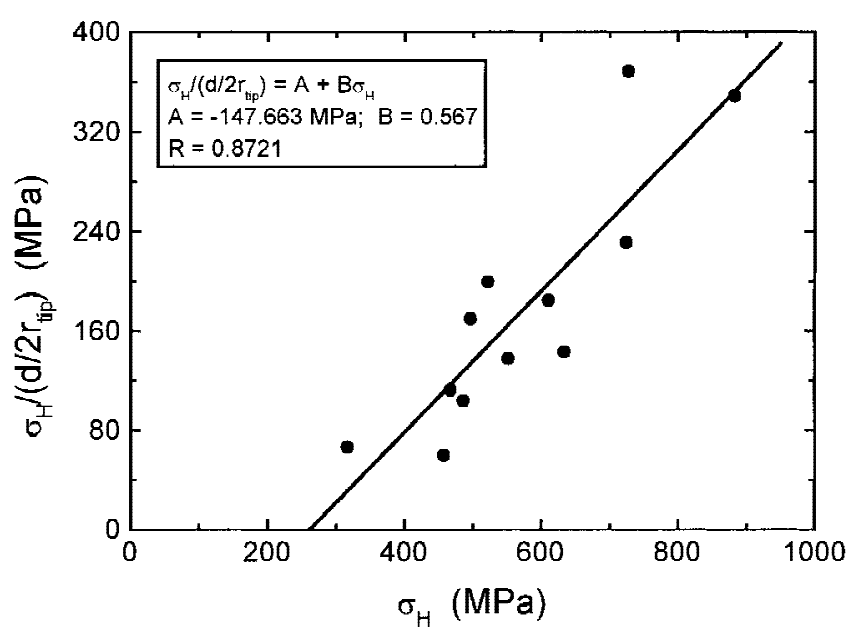

FIG. 8. Dependence of hardening coefficient $\theta=\sigma_{\mathrm{H}} /\left(a / 2 R_{\text {tip }}\right)$ on hardness stress $\sigma_{\mathrm{H}}$.

also finds that $\sigma_{\mathrm{Hs}}=1.8 \times 10^{-3} G$ (where the shear modulus $G=148 \mathrm{GPa}$ for $\mathrm{MgO}$ ). Interestingly, the ratio $\sigma_{\mathrm{Hs}} / G$ agrees well with the value of approximately $5 \times$ $10^{-3}$ in stage III for face-centered-cubic metals. ${ }^{41}$

From the above, it may be concluded that the decrease in nanobardness $\sigma_{\mathrm{H}}$ with increasing indentation size $a$ is a result of strain softening process. The observed absence of slip lines around indentation impressions suggests that the softening process involves redistribution of point defects produced during deformation.

\section{DEPENDENCE OF HILL HEIGHT ON OTHER INDENTATION PARAMETERS}

\section{A. Indentation hills and Johnson's cavity model}

From an atomic force microscopy study, Harvey et al. ${ }^{17}$ reported that the displacement of material as hills around indents on a surface may be described by Johnson's cavity model and Lockett's model based on the slip-line theory for rigid plastic materials. These authors found that Johnson's cavity model predicts satisfactorily the profiles of the dependence of magnitudes of $h$ on distance $r$ from the indent center. Here we compare the experimental data on the formation of hills around indents on $\mathrm{MgO}$ with the predictions of the cavity model.

The relationship between the displacement $h$ of the material piled up as a hill, the indentation diameter $a$, and the deformation zone radius $R$ is given by ${ }^{17}$

$$
\frac{h(a)}{a}=\frac{2 \pi(1-v) Y}{E^{*}}\left(\frac{R^{3}}{a^{3}}-1 / 8\right),
$$

where we have substituted indentation diameter $a=2 r$ for the indentation radius $r$ in the original equation of Ref. 17 and the combined elastic modulus $E^{*}$ is given by Eq. (2). In principle, this equation is valid only for $R / a \geq 1 / 2$ (i.e., when $h(a) / a \geqslant 0$ ).
Substitution of the values of $E^{*}=115 \mathrm{GPa}$ (Sec. III. A), $Y=80 \mathrm{MPa}^{32}$ and $v=0.3$ in Eq. (10) yields

$$
h=3.06 \times 10^{-3} a\left(\frac{R^{3}}{a^{3}}-1 / 8\right) \quad .
$$

Since $R / a$ is practically a constant quantity, hill height $h(\mathrm{a})$ increases linearly with $a$ with a slope $3.06 \times$ $10^{-3}\left[(R / a)^{3}-1 / 8\right]$.

The slope of the $h / a$ plot from the experimental data is 0.078 , which gives the ratio $R / a=2.95$. This $R / a$ ratio is higher by a factor of 1.8 than the ratio from the experimental data shown in Fig. 4. This difference suggests that Johnson's cavity model relating $h$ and $a$ in the form of Eq. (11) overestimates $R$ (because $a$ can be measured with greater accuracy).

\section{B. Indentation crater model}

According to this model, the material of the indentation cavity of volume $V_{\text {ind }}$ is forced out from the crystal to form a uniform elevated rim of volume $V_{\text {rim }}$ around the cavity. The hill height may be given by (see the Appendix)

$$
h=\frac{2 d}{3 \pi\left[(2 R / a)^{2}-1\right]} .
$$

Since $R / a$ as a constant in the present case, $h$ linearly increases with $d$. Equation (12) is valid for $R / a>1 / 2$ when $h<\infty$. The linear dependence of $h$ on $d$ may be observed from Fig. 1.

The slope of $h(d)$ plot is $\left.2 / 3 \pi\left[(2 R / a)^{2}-1\right)\right]=0.071$ (see Table I), which gives $R=a$. Thus, this model reveals that the experimentally measured $R$ values in our case are higher by a factor of 1.67. This means that, in contrast to Johnson's model [Eq. (11)] which overestimates $R$, Eq. (12) underestimates $R$. This discrepancy is probably due to higher values of $R$ and lower values of $h$ in Eq. (12) than the values measured from experiments.

\section{CONCLUSIONS}

From the present study on the nanoindentation of (100) face of $\mathrm{MgO}$ crystals at loads up to $10 \mathrm{mN}$, the following conclusions can be drawn:

(1) Indentation diameter $a$, radius $R$ of plastic zone, and the height $h$ of hills piled up around indents linearly increase with the indentation penetration depth $d$. As suggested by the two threshold values for the penetration depth, the indentation involves two different processes. The first is associated with the development of indentation size and deformation zone (threshold penetration $d_{0}=29.7 \mathrm{~nm}$ ), while the second is associated with the formation of hills which appear only when $d>d_{0}$.

(2) A comparison of various models, considered in this work to analyze the experimental dependence of deformation parameters $a, R$, and $d$ on indentation load, 
shows that Johnson's cavity model of elastic-plastic boundary explains the experimental data satisfactorily. Tabor's model for plastic materials, Sneddon's model for perfect elastic materials, classical Hertzian theory, and Hertzian theory for perfect elastic contacts are not valid during the nanoindentation of $\mathrm{MgO}$.

(3) Nanoindentation hardness of the (100) face of $\mathrm{MgO}$ crystals is lower by a factor of 10-25 than those during microhardness measurements while its loadindependent nanohardness is $294 \mathrm{MPa}$. This large discrepancy between nano- and microhardness is due to the assumption that the ratio of hardness $H$ to yield stress $Y$ is 3 instead of 35 for many single crystals undergoing plastic deformation.

(4) Indentation deformation of $\mathrm{MgO}$ crystals at low loads represents a typical example of the load dependence of hardness representing normal indentation size effect (i.e., hardness increases with a decrease in load) and is associated with the strain-softening of the material during indentation. However, in the range of loads used in the indentation experiments here, no evidence of plastic deformation of $\mathrm{MgO}$ crystals was observed.

(5) The experimental data on the interdependence between the hill height $h$, the indentation diameter $a$, and the deformation zone radius $R$ are satisfactorily described by Johnson's cavity model. A new model based on the concept of displacement and piling up of indented material (indentation crater model) is also found to interpret the experimental data well. However, Johnson's cavity model somewhat overestimates while the indentation crater model somewhat underestimates the value of the radius of deformed zone.

\section{ACKNOWLEDGMENTS}

The authors express their sincere gratitude to the Serveis Cientifico-Tecnics of the University of Barcelona for technical facilities. One of the authors (K.S.) also expresses his gratefulness to the authorities of the University of Barcelona for extending an invitation to work as a visiting professor during the academic year 1995-1996 and to the Generalitat de Catalunya for granting him a maintenance grant.

\section{REFERENCES}

1. R.W. Mott, Microindentation Hardness Testing (Butterworths, London, 1956).

2. P.M. Sargent, in Microindentation Techniques in Materials Science and Engineering ASTM STP 889, edited by P.J. Blau and B.R. Lawn (ASTM, Philadelphia, PA, 1985), p. 165.

3. W.C. Oliver, R. Hutchings, and J.B. Pethica, in Microindentation Techniques in Materials Science and Engineering ASTM STP 889, edited by P.J. Blau and B.R. Lawn (ASTM, Philadelphia, PA, 1985), p. 90.
4. J.L. Loubet, J.M. Georges, and G. Meille, in Microindentation Techniques in Materials Science and Engineering ASTM STP 889, edited by P.J. Blau and B.R. Lawn (ASTM, Philadelphia, PA, 1985), p. 72.

5. S.J. Bull, T.F. Page, and E.H. Yoffe, Philos. Mag. Lett. 59, 281 (1989).

6. H. Li, A. Ghosh, Y.H. Hau, and R.C. Bradt, J. Mater. Res. 8, 1028 (1993).

7. H. Li and R.C. Bradt, J. Mater. Sci. 28, 917 (1993).

8. H. Li, Y.H. Han, and R.C. Bradt, J. Mater. Sci. 29, 5641 (1994).

9. K. Sangwal, J. Mater. Sci. 24, 1128 (1989).

10. K. Sangwal, Mater. Chem. \& Phys. (in press).

11. Q. Ma and D.R. Clarke, J. Mater. Res. 10, 853 (1995).

12. Ch. Ullner and L. Höhne, Phys. Status Solidi A 129, 167 (1992).

13. Yu.S. Boyarskaya, D.Z. Grabko, and E.I. Purich, J. Mater. Sci. 14, 737 (1979).

14. J. Guille and M. Sieskind, J. Mater. Sci. 26, 899 (1991).

15. G.M. Pharr, W.C. Oliver, and D.S. Harding, J. Mater. Res. 6, 1129 (1991).

16. T.F. Page, W.C. Oliver, and C.J. McHargue, J. Mater. Res. 7, 450 (1992).

17. S.E. Harvey, H. Huang, S.K. Venkataraman, and W.W. Gerberich, J. Mater. Res. 8, 1291 (1993).

18. W. Zielinski, H. Huang, and W.W. Gerberich, J. Mater. Res. 8, 1300 (1993).

19. E.T. Lilleodden, W. Bonin, J. Nelson, J.T. Wyrobek, and W.W. Gerberich, J. Mater. Res. 10, 2162 (1995).

20. E.R. Weppelmann, J.S. Field, and M.V. Swain, J. Mater. Res. 8, 830 (1993)

21. J.J. Gilman, J. Mater. Res. 7, 535 (1992).

22. I.G. Berzina, I.B. Berman, and P.A. Savintsev, Kristallografiya 9, 483 (1965).

23. V.P. Bhatt, R.M. Patel, and C.F. Desai, Cryst. Res. Technol. 18, 1173 (1983).

24. K. Sangwal and S.K. Arora, J. Mater. Sci. 13, 1977 (1978).

25. K. Sangwal and S.K. Arora, J. Phys. D: Appl. Phys. 12, 645 (1979).

26. C.A. Brookes, R.P. Burnand, and J.E. Morgan, J. Mater. Sci. 10, 2171 (1975).

27. E. Meyer and H. Heinzelmann, in Scanning Tunneling Microscopy II, edited by R. Wiesendanger, and H-J. Gunlherodt (Springer-Verlag, Heidelberg, Germany, 1992), p. 99.

28. C. Kittel, Introduction to Solid State Physics, 5th ed. (Wiley, New York, 1976), Chap. 4.

29. D. Tabor, The Hardness of Metals (Clarendon Press, Oxford, United Kingdom, 1951).

30. D. Tabor, in Microindentation Techniques in Materials Science and Engineering ASTM STP 889, edited by P.J. Blau and B.R. Lawn (ASTM, Philadelphia, PA, 1985), p. 129.

31. K.L. Johnson, Contact Mechanics (Cambridge University Press, Cambridge, United Kingdom, 1985).

32. P.J. Stablein, J. Appl. Phys. 34, 1867 (1963).

33. B.L. Hammond and R.W. Armstrong, Philos. Mag. 57, 41 (1988).

34. C. Hays and E.G. Kendall, Metallurgy 6, 275 (1973).

35. N.A. Stelmashenko, M.G. Walls, L.M. Brown, and Yu. V. Milman, Acta Metall. Mater. 41, 2855 (1993).

36. N.A. Fleck, G.M. Muller, M.F. Ashby, and J.W. Hutchinson, Acta Metall. Mater. 42, 475 (1994).

37. J.H. Westbrook, Proc. Am. Soc. Testing Mater. 57, 873 (1957).

38. K. Sangwal, P. Gorostiza, and F. Sanz, Surf. Sci. (in press).

39. N. Gane, Proc. R. Soc. London A317, 367 (1970).

40. A. Gerk, J. Mater. Sci. 12, 735 (1977).

41. J. Gil Sevillano, in Plastic Deformation and Fracture of Materials, edited by H. Mughrabi (VCH, Weinheim, Germany, 1993), p. 19. 


\section{APPENDIX: INDENTATION CRATER MODEL}

The main idea of this model is that the material of the indentation cavity is forced out from the crystal to form a uniform elevated rim around the cavity (Fig. A1). If the density of the piled up material in the rim is equal to that of the crystalline solid, the volume $V_{\text {rim }}$ of the piled rim is equal to the volume $V_{\text {ind }}$ of the indentation cavity.

Since at moderate loads the observed indentation cavities are conical, we consider the case of conical indentation. If this cavity has a depth $d$ and diameter $a$ with respect to the indented surface, the volume $V_{\text {ind }}$ of the indentation cavity may be given by

$$
V_{\text {ind }}=(\pi / 12) a^{2} d \text {. }
$$

Let us assume that in cross section the arc-shaped rim has a base of width $\delta$ and a maximum height $h$ while its circumference around the cavity at the point of maximum height is $l=\pi(a+\delta)=\pi(R+a / 2)$. Then the volume of the rim may be given by (see Fig. A1)

$$
\begin{aligned}
V_{\text {rim }} & =(\pi / 2) l D h \cos \phi \\
& =\left(\pi^{2} / 2\right) D h(R+a / 2) \cos \phi .
\end{aligned}
$$

With reference to point $\mathrm{A}$ in the circle of Fig. A1, one obtains

$$
D \cos \phi=\delta=R-a / 2 .
$$

Substituting this value of $D \cos \phi$ in Eq. (A2), one gets

$$
\begin{aligned}
V_{\text {rim }} & =\left(\pi^{2} / 2\right) h(R+a / 2)(R-a / 2) \\
& =\left(\pi^{2} / 2\right) h\left(R^{2}-a^{2} / 4\right) .
\end{aligned}
$$

Taking $V_{\text {ind }}=V_{\text {rim }}$, from Eqs. (A1) and (A4) one may express the hill (rim) height in the form

$$
h=\frac{2 d}{3 \pi\left[(2 R / a)^{2}-1\right]} .
$$

This is a general expression relating hill height $h$ with other measureable parameters like indentation depth $d$, indentation diameter $a$, and radius $R$ of the deformed zone.

It may be seen that Eq. (A5) is valid for $R>a / 2$ when $h<\infty$ and $h$ decreases with increasing $R / a$. However, small values of $h$ do not affect the values of $R / a$ appre- ciably whereas small changes in $R / a$ lead to enormous changes in $h$. Immediately after indentation if a hill starts flowing (spreading) laterally on the indented surface immediately after indentation, it should lead to an increase in the width of the hill rim (i.e., increase in $R$ ) as well as a decrease in its height $h$. In this case the lower experimental $h(d)$ values are expected to give the right $R / a$ values. It is also possible that the rim volume $V_{\text {rim }}$ is higher than $V_{\text {ind }}$ by a few percent due to introduction of point defects in the material displaced from the indent. This results in an increase in both $h$ and $R$. In this case the higher experimental $h(d)$ values are expected to give $R / a$ values which are higher than those predicted from Eq. (A5). Thus, according to Eq. (A5), depending on whether the lateral spreading process or the increase in the volume of the material displaced by the indenter is predominant, the dependence of hill height $h$ on indentation depth $d$ is expected to give $R / a$ values which can be higher or lower than the experimental values.

Usually, the value of $2 R / a$ is approximately a constant quantity. Consequently, the $h(d)$ dependence is linear. Similarly, when the ratio a/d is a constant as in the present case, $h(a)$ is also linear. However, in time-dependent processes like indentation creep and indentation recovery, where the dependences of $a$ (also $R$ ) and $d$ on the time of relaxation after the application of a particular load are different, $h(d)$ dependence may not be linear. In such cases it is better to use the general expression (A5).

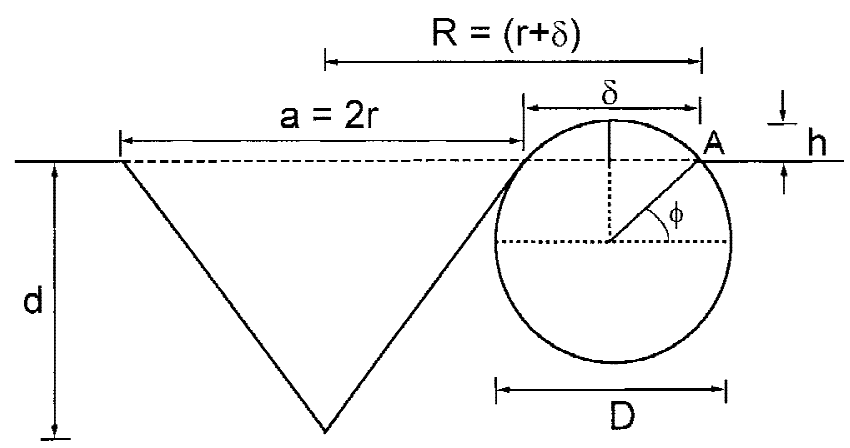

FIG. A1. Schematic illustration of the indentation crater model. The material of the indentation cavity is piled up as a circular elevated arc-shaped rim in cross section. The width of the base of the rim is $\delta$, and the maximum height is $h$. 